\title{
SADLABI: Proposta de um Sistema de Apoio à Decisão para a gerência de Laboratórios de Informática
}

\author{
Cristovão S. Lima, Hélio E. M. Júnior, Soraya R. S. Medeiros, \\ Angélica F. Medeiros
}
Bacharelado em Sistemas de Informação - Centro de Ensino Superior do Seridó (CERES/UFRN) - Caixa Postal - 59300-000 - Caicó - RN - Brasil
\{cristovaolima, helio_junior, soraya_rb\}@ufrn.edu.br, angelicafelixx@gmail.com

\begin{abstract}
The present work analyzes the open data on the access, use and appropriation of ICTs in Brazilian schools provided by CETIC and proposes the creation of a Decision Support System to assist in the decision process regarding the application of ICTs in Brazilian schools. To do so, this research aims to outline the necessary requirements for system development, as well as system models and screen prototypes that will help in the development of DSS. As a result, the artifacts that have been developed are presented.
\end{abstract}

Resumo. O presente trabalho analisa os dados abertos sobre o acesso, uso e apropriação das TICs nas escolas do Brasil fornecidos pela CETIC e propõe a criação de um Sistema de Apoio à Decisão para auxiliar no processo de decisão referente a aplicação das TICs nas escolas brasileiras. Para tanto, esta pesquisa tem como objetivo traçar os requisitos necessários para o desenvolvimento do sistema, além de modelos de sistema e protótipos de tela que irão ajudar no desenvolvimento do SAD. Como resultado, são apresentados os artefatos que foram desenvolvidos.

\section{Introdução}

O uso de Tecnologias da Informação e Comunicação (TICs) na Educação, em seus mais variados níveis, é apontado como fortalecedor para o processo de ensino-aprendizagem [Toledo 2015], tanto para o docente, pois sua prática se torna mais lúdica, quanto para o discente, visto que permite que exista o complemento entre os saberes compartilhados em sala de aula e os abordados fora dela por meio de ferramentas, como o computador, em laboratórios de informática [Pereira et al. 2018].

Em decorrência desses fatores, vários investimentos foram realizados com o objetivo de implantar Laboratórios de Informática (LI) em escolas públicas. Em uma breve revisão, encontra-se o Programa Nacional de Tecnologia Educacional (ProInfo), Programa Educacional criado pela Portaria $\mathrm{n}^{\circ}$ 522/MEC, de 9 de abril de 1997, com o intuito de promover o uso pedagógico das TICs na rede pública de ensino fundamental e médio, assim, o Governo Federal é o responsável por prover às escolas computadores, recursos digitais e conteúdos educacionais, enquanto que os Estados e municípios realizam a gestão desses espaços.

No entanto, diversas problemáticas surgem quando volta-se a ótica para essa gestão e relacionado a isto, existem apenas considerações empíricas que se sobressaem 
VIII Congresso Brasileiro de Informática na Educação (CBIE 2019)

Anais do XXV Workshop de Informática na Escola (WIE 2019)

acerca da realidade dos LI. Além disso, existem outras questões que devem ser analisadas para que o Setor Público compreenda o que é a realidade desses espaços, com vistas a melhorar o gerenciamento desses laboratórios e destinar de forma mais racional no que de fato precisa melhorar nesses locais.

Para que se possa atingir tal propósito, uma das possibilidades é a utilização de um Sistemas de Apoio à Decisão (SAD) aliado ao uso de dados abertos com o objetivo de ajudar na tomada de decisão, pois permite que se tenha a facilidade de acessar vários dados e minerá-los com filtros, de acordo com o que a necessidade do gestor, para que sejam tomadas decisões mais coerentes.

Neste sentido, o presente trabalho propõe um Sistema de Apoio à Decisão (SAD), que faz uso de dados abertos governamentais, para auxiliar o processo decisório do uso dos laboratórios de informáticas e cursos com base nos dados disponibilizados pelo site CETIC $^{1}$ sobre o uso e apropriação das TICs nas escolas do Brasil. Para tanto, ele será do tipo dashboard, onde terão gráficos, que serão gerados com base nos dados abertos para apoiar processo de decisão.

\section{Trabalhos relacionados}

Nesta pesquisa serão enfatizados os trabalhos de [Magalhães and Cardoso 2017] e [Santos et al. 2017] pois abordam e trazem resultados mais relacionados ao proposto neste. O estudo de [Magalhães and Cardoso 2017] constrói um modelo de Análise de Dados Abertos sobre o Ensino Superior Brasileiro para a geração de relatórios analíticos (Dashboards) com o objetivo de auxiliar no processo de tomada de decisão. Como resultado, apresentaram dados sobre o quantitativo de alunos formados por região, quantitativo de alunos matriculados por área de conhecimento entre outros.

[Santos et al. 2017] tiveram como objetivo realizar um mapeamento sistemático levando em consideração o período de 2008-2017 e apontaram que apenas a partir de 2011 foram localizados artigos relacionados a área estudada. E dentre os resultados obtidos, identificaram a demanda de sistemas para apoiar o gestor na tomada de decisão; predição de desempenho de escolas.

Com isso, destaca-se que, embora muitas pesquisas apliquem dados abertos à educação, pouco se tem em torno destes com o propósito de facilitar ou potencializar a gestão dos laboratórios. Além disso, este trabalho se diferencia dos demais, por propor um SAD e não somente um modelo de análise de dados, destacando a importância de desenvolver soluções através desta intervenção.

\section{Metodologia}

Metodologicamente o trabalho foi dividido em quatro etapas: i) realizar revisão literária; ii) identificar e especificar requisitos; iii) projetar e modelagem do sistema; iv) desenvolver os protótipos. Para cumprir a primeira etapa, foi realizada uma revisão literária sobre SAD e dados abertos, modelos e protótipos de sistema. Com isso, foi desenvolvido a documentação do sistema que descreve as funcionalidades do mesmo. Para tanto, foi elaborado o documento de requisitos de software [Sommerville 2011] para especificar e detalhar a problemática do sistema proposto.

\footnotetext{
${ }^{1}$ https://cetic.br/pesquisa/educacao/
} 
VIII Congresso Brasileiro de Informática na Educação (CBIE 2019)

Anais do XXV Workshop de Informática na Escola (WIE 2019)

Como modelos do sistema foram escolhidos os Diagramas de Casos de Uso e de Classes com o objetivo de esclarecer e ajudar na compreensão do problema. Tais modelos têm o objetivo de permitir o entendimento de maneira simples e visual das classes utilizadas, com orientação a objetos e dos atores que irão utilizar o sistema com suas respectivas funcionalidades. Para desenvolver os modelos foi utilizada a ferramenta Draw.io ${ }^{2}$ e para desenvolver os protótipos de tela foi utilizada a ferramenta Figma ${ }^{3}$.

\section{Resultados e discussões}

O presente trabalho propõe a criação de uma sistema de apoio à decisão onde os dados sobre as TICs na educação, fornecidos pelo CETIC, serão filtrados e apresentados em Dashboards para auxiliar o processo decisório do uso dos laboratórios de informática e cursos. Neste sentido, o SAD objetiva que a partir desses dados, seja possível mapear os laboratórios de informática existentes nas escolas públicas brasileiras para decidir quais critérios serão levados em consideração para melhorar a administração desses espaços. Além disso, estabelecer de maneira mais eficiente os problemas que precisam ser resolvidos quanto ao uso dos laboratórios de informática da rede pública e municipal de ensino. A partir disso, foram definidos os requisitos necessários para o desenvolvimento do SAD.

O sistema comporta o requisito Funcional: CRUD de dados. Este requisito se refere ao cadastro, atualização e exclusão dos dados do sistema que serão inseridos no banco de dados com base nos dados fornecidos pelo CETIC. A partir dos dados cadastrados o usuário poderá aplicar filtros para criar dashboards com as métricas que achar necessário. Os requisitos Não-Funcionais especificados foram: usabilidade, desempenho, repositório de códigos, implementação, responsividade, confiabilidade, padrões, interoperabilidade e ética.

\subsection{Modelos do sistema}

Os modelos do sistemas definidos para auxiliar no desenvolvimento foram os Diagramas de Classes e Casos de uso, com o objetivo de esclarecer e evitar redundâncias na compreensão do problema. O Diagrama de Casos de Uso demonstra o ator usuário e as funcionalidade que o mesmo pode desempenhar no SAD, que seria, importar os dados XML para a aplicação, escolher os filtros para gerar os gráficos. A seguir está a Figura 1 que o representa.

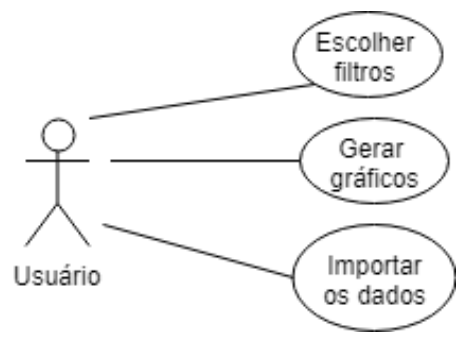

Figura 1. Casos de uso

\footnotetext{
${ }^{2}$ O Draw.io é uma ferramenta online para a criação de Diagramas, gráficos e outros sem a necessidade de usar um software caro e pesado.

${ }^{3}$ O Figma é uma software online para prototipação de telas, permite a criação de telas mais reais e com navegabilidade.
} 
VIII Congresso Brasileiro de Informática na Educação (CBIE 2019)

Anais do XXV Workshop de Informática na Escola (WIE 2019)

O Diagrama de Classes desenvolvido possui dois pacotes, o MODEL e o DAO. No pacote MODEL estão representadas as classes/objetos do sistema e os seus atributos. Já o DAO representa as classes de controle, onde ficam todos os métodos de ações que o usuário poderá realizar. Existe uma ligação entre os pacotes partindo do DAO, ele tem a responsabilidade de controlar as classes. A Figura 2 mostra o Diagrama desenvolvido a partir da problemática apresentada.

DAO

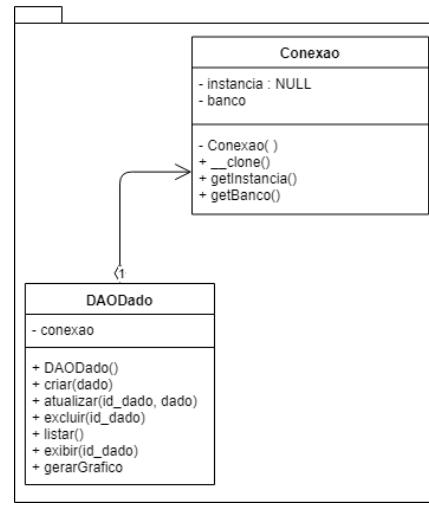

MODEL

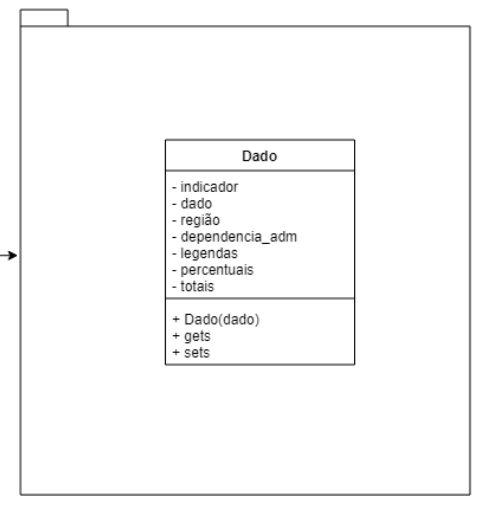

Figura 2. Diagrama de classes

A partir dos dados abertos disponibilizados pelo CETIC, será gerado um arquivo do tipo Extensible Markup Language (XML), esse tipo de arquivo é mais vantajoso pois ele possibilita atribuir tags para identificar as informações além de serem armazenados em formato de texto, o que facilita na hora de atualizações. O arquivo XML contendo os dados será salvo no banco e, a partir dele, o usuário poderá filtrar as informações.

\subsection{Protótipos de tela}

Para melhor compreensão de como será a interface do sistema proposto, foram desenvolvidos os protótipos de alta fidelidade. Na Figura 3, será apresentado o protótipo da tela principal do sistema.

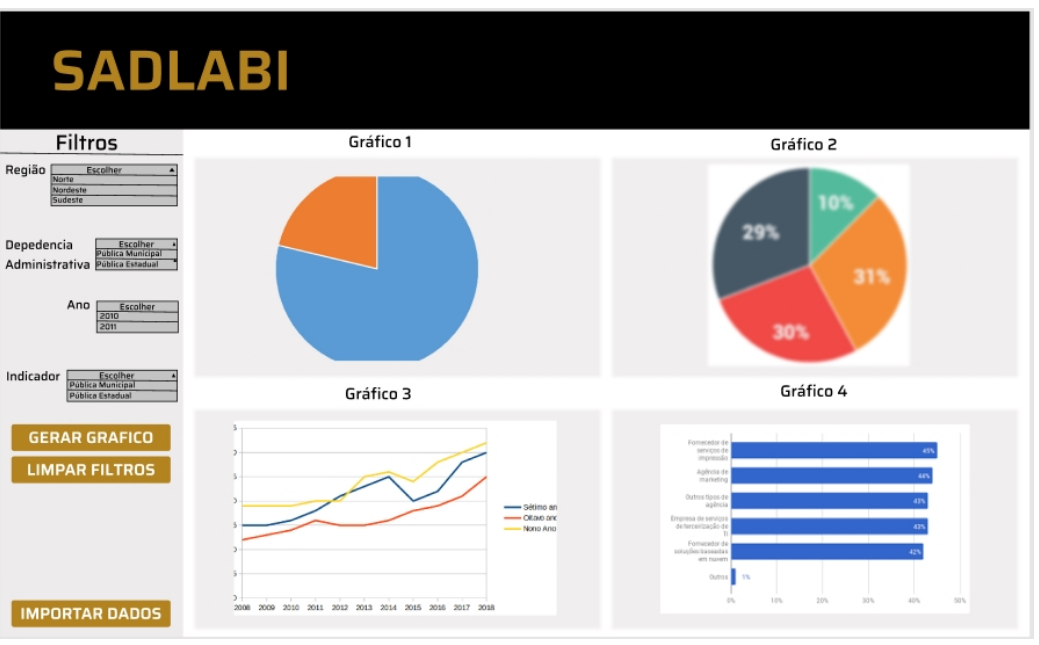

Figura 3. Tela home SadLabi 
VIII Congresso Brasileiro de Informática na Educação (CBIE 2019)

Anais do XXV Workshop de Informática na Escola (WIE 2019)

No canto esquerdo, o usuário tem as opções de filtros e importar os dados para o SAD. Ele poderá filtrar por região, dependência administrativa, ano e indicador. A partir do filtros escolhidos ele clica no botão de gerar gráfico, quatro modelos de gráficos serão gerados a partir do dados que ele escolheu.

\section{Conclusão}

Neste artigo foi possível discutir sobre o uso de TICs e a sua influência no processo de ensino e aprendizagem, bem como, observar o incentivo governamental, por meio do PROINFO, para a propagação de ferramentas, tais como os computadores e os laboratórios de informática, a fim de gerar inclusão digital para os alunos em diversos estados brasileiros. Além disso, percebeu-se que não adianta inserir os computadores, sem que haja um gerenciamento mais rigoroso por meio dos estados e municípios, os responsáveis, consoante à Lei, por esse processo, isso porque esses espaços, se não bem aproveitados, podem se tornar obsoletos e serem depósitos de desperdício de dinheiro público.Neste cenário se insere a problemática que foi trabalhada por este estudo, tal como, através de dados abertos governamentais, propor e realizar um maior controle dos laboratórios de informática. Assim, neste trabalho foi desenvolvida a documentação de um Sistema de Apoio à Decisão que utiliza a base de dados abertos para expor aos gestores, de que forma aqueles espaços estão sendo utilizados, a fim de que o dinheiro público seja mais bem direcionado. Como trabalhos futuros, objetiva-se modelar o banco de dados do sistemas de forma a atender a necessidade de manipulação dos dados pelo usuário, e também implementar o SAD, realizar avaliações sobre a sua usabilidade e aplicá-los em cenários reais.

\section{Referências}

Magalhães, H. F. and Cardoso, L. d. A. (2017). Análise de dados abertos sobre o ensino superior brasileiro.

Pereira, C. H. S. et al. (2018). Utilização do laboratório de informática no processo de ensino e aprendizagem de matemática.

Santos, P., Ferreira, R., and Miranda, P. (2017). Dados abertos educacionais: Uma revisao da literatura brasileira. In Brazilian Symposium on Computers in Education (Simpósio Brasileiro de Informática na Educação-SBIE), volume 28, page 11.

Sommerville, I. (2011). Engenharia de Software. 9. Pearson Prentice Hall, São Paulo.

Toledo, B. S. (2015). O uso de softwares como ferramenta de ensino-aprendizagem na educação do ensino médio/técnico no instituto federal de minas gerais. Projetos $e$ Dissertações em Sistemas de Informação e Gestão do Conhecimento, 4(2). 